Pacific

Journal of

Mathematics

EISENSTEIN PRIMES, CRITICAL VALUES AND GLOBAL TORSION

NeIL Dummigan

Volume 233 No. 2

December 2007 


\title{
EISENSTEIN PRIMES, CRITICAL VALUES AND GLOBAL TORSION
}

\author{
NEIL DUMMIGAN
}

\begin{abstract}
We consider congruences between Eisenstein series and cusp forms - of weight $k$, level $N$ and character $\chi$ of conductor $N$ - modulo large prime divisors of $L\left(1-k, \chi^{-1}\right)$. We show that such primes occur in the order of a "global torsion" group attached to the cusp form $f$, and (under a certain hypothesis) also in the denominator of the algebraic part of the rightmost critical value $L_{f}(k-1)$. These occurrences are linked by the Bloch-Kato conjecture.
\end{abstract}

\section{Introduction}

We set $\kappa(k):=(-1)^{k}$ throughout. Let $f$ be a normalised newform in $S_{k}\left(\Gamma_{1}(N), \chi\right)$. Here $k \geq 2$ and $N \geq 1$ are integers, $\chi:(\mathbb{Z} / N \mathbb{Z})^{\times} \rightarrow \mathbb{C}^{\times}$is a character, and

$$
\Gamma_{1}(N)=\left\{\left[\begin{array}{ll}
a & b \\
c & d
\end{array}\right] \in \mathrm{SL}_{2}(\mathbb{Z}) \mid c \equiv 0 \quad(\bmod N), d \equiv 1 \quad(\bmod N)\right\} .
$$

If

$$
\Gamma_{0}(N)=\left\{\left[\begin{array}{ll}
a & b \\
c & d
\end{array}\right] \in \mathrm{SL}_{2}(\mathbb{Z}) \mid c \equiv 0 \quad(\bmod N)\right\}
$$

then the holomorphic function $f$ on the complex upper half plane satisfies

$$
f\left(\frac{a \tau+b}{c \tau+d}\right)=\chi(d)(c \tau+d)^{k} f(\tau) \quad \text { for all }\left[\begin{array}{ll}
a & b \\
c & d
\end{array}\right] \in \Gamma_{0}(N) .
$$

Since $\left[\begin{array}{rr}-1 & 0 \\ 0 & -1\end{array}\right] \in \Gamma_{0}(N)$ and $f \neq 0$, necessarily $\chi(-1)=(-1)^{k}$. The Fourier expansion of $f$ is of the form $f(\tau)=\sum_{n=1}^{\infty} a_{n} q^{n}$ with $a_{1}=1$, where $q=e^{2 \pi i \tau}$. The $a_{n}$ lie in the ring of integers of some finite extension of $\mathbb{Q}$, and each Hecke operator $T_{n}$ satisfies $T_{n} f=a_{n} f$.

The $L$-series $L_{f}(s)=\sum_{n=1}^{\infty} a_{n} n^{-s}$ converges for $\Re(s)>(k+1) / 2$ and has an Euler product. It defines a function with an analytic continuation to the whole

MSC2000: 11F67, 14G10.

Keywords: modular form, L-function, Bloch-Kato conjecture. 
complex plane, with

$$
(2 \pi)^{-s} \Gamma(s) L_{f}(s)=\int_{0}^{\infty} f(i y) y^{s} \frac{d y}{y} .
$$

In fact $L_{f}(s)$ is the $L$-function attached to a premotivic structure; see [Diamond et al. 2004, 1.1.1] for precise definitions. At precisely the points $s=1, \ldots, k-1$, it is critical in the sense of [Deligne 1979]. As in [Section 7] there, the above integral expression for $L_{f}(s)$ enables one to verify the relevant case of Deligne's conjecture, which interprets the critical values, up to algebraic multiples, as certain periods. The relevant case of the Bloch-Kato conjecture [1990] removes the ambiguity about the algebraic multiple, up to a unit. It predicts that, for $1 \leq j \leq k-1$,

$$
\frac{L_{f}(j)}{(2 \pi i)^{j} \Omega^{\kappa(j)}}=\frac{\prod_{p \leq \infty} c_{p}(j) \# \amalg(j)}{\# H^{0}(\mathbb{Q}, A(j)) \# H^{0}(\mathbb{Q}, \check{A}(1-j))} .
$$

The various terms will be defined in Section 3, but this should be viewed as analogous to the rank 0 case of the formula of Birch and Swinnerton-Dyer.

For a character $\chi$ of conductor $N$, consider the Eisenstein series $E_{k}^{\chi, 1}$. (We must exclude the case $k=2, N=1$.) This noncusp form belongs to $M_{k}\left(\Gamma_{1}(N), \chi\right)$, and $T_{p}\left(E_{k}^{\chi, 1}\right)=\left(\chi(p)+p^{k-1}\right) E_{k}^{\chi, 1}$ for every prime $p$. If $\lambda \nmid 6 N k$ ! divides the Dirichlet $L$-value $L\left(1-k, \chi^{-1}\right)$, we show, in Section 2, that there is a newform $f \in S_{k}\left(\Gamma_{1}(N), \chi\right)$ with $f \equiv E_{k}^{\chi, 1}(\bmod \lambda)$ (as Fourier expansions). Here $\lambda$ is a prime divisor for a number field $K$ large enough to contain the values of $\chi$ and the Fourier coefficients of $f$. (Actually, Proposition 2.1 deals with a somewhat more general type of Eisenstein series.) Congruences of this type are well known. The case $k=12, N=1$ is Ramanujan's congruence $\tau(n) \equiv \sigma_{11}(n)(\bmod 691)$. The case $k=2, N=p, \lambda \mid p$ (not satisfying our condition $\lambda \nmid N$ ) was used by Ribet [1976].

The various terms in (1) depend on a choice of " $S$-integral premotivic structure", though the ratio of the two sides is independent of the choice. We make a natural choice as in [Diamond et al. 2004]. Having done this, there is a 2-dimensional $\mathbb{F}_{\lambda}$-vector space $A[\lambda]$ with $\operatorname{Gal}(\overline{\mathbb{Q}} / \mathbb{Q})$-action, which may be viewed as analogous to (a twist of) the group of $\ell$-torsion points on an elliptic curve. It follows from the fact that (for all primes $p) a_{p} \equiv \chi(p)+p^{k-1}(\bmod \lambda)$ that $A[\lambda]$ is reducible, with composition factors $\mathbb{F}_{\lambda}\left(\chi^{-1}\right)$ and $\mathbb{F}_{\lambda}(1-k)$. If the latter is a submodule, then $A[\lambda](k-1)$ has a trivial submodule. This would contribute to the $\lambda$-part of $\# H^{0}(\mathbb{Q}, A(k-1))$ in the denominator of (1) (in the case $\left.j=k-1\right)$. In [Dummigan 2000], I speculated that this is the case, and, following the proof of [Faltings and Jordan 1995, Theorem 4.6], I proved it [Dummigan 2005, Theorem 7.3] in the case $N=1, K=\mathbb{Q}$, which probably means just $k=12, \ell=691 ; k=16, \ell=3617 ; k=18$, $\ell=43867 ; k=20, \ell=283$ or $617 ; k=22, \ell=131$ or 593 ; and $k=26, \ell=657931$. 
In Section 4, we see that the proof carries across easily to the more general case considered here. Moreover, we show that the $\lambda$-parts of the Tamagawa factors $c_{p}(k-1)$, which appear in the numerator, are trivial, even for $p \mid N$. Hence, unless there is nontrivial $\lambda$-torsion in $\amalg(k-1)$, we expect to see $\lambda$ in the denominator of $L_{f}(k-1) /\left((2 \pi i)^{k-1} \Omega^{\kappa(k-1)}\right)$.

In [Dummigan 2005, Section 8] we noted that this can be observed in Stein's [ $\geq 2008$ ] numerical data in the case $N=1, K=\mathbb{Q}$. In [Dummigan 2000], we saw the related (but weaker) fact that (again in the case $N=1, K=\mathbb{Q}$ ) $\lambda=\ell$ appears in various "period ratios", which are essentially ratios of critical values of $L_{f}(s)$. This was observed numerically, using data of Manin [1973], and also proved theoretically, using a formula of Kohnen and Zagier [1984]. In Sections 5, 6 , and 7, we give a proper proof and explanation, in the general case, of why $\lambda$ appears in the denominator of $L_{f}(k-1) /\left((2 \pi i)^{k-1} \Omega^{\kappa(k-1)}\right)$. We have to impose the condition that $\lambda$ is not a congruence prime for $f$ in $S_{k}\left(\Gamma_{1}(N), \chi\right)$; see before Lemma 6.2.

The proof uses the well-established principle that modular symbols provide a bridge between the cohomology of modular curves (with coefficients in appropriate local systems) and critical values of modular $L$-functions. To relate congruences of modular forms to congruences of cohomology classes, we make essential use, largely via [Diamond et al. 2004], of the Fontaine-Lafaille integral theory of crystalline representations, and of Faltings's comparison theorem.

It is natural to ask whether the condition that $\lambda$ should not be a congruence prime for $f$ in $S_{k}\left(\Gamma_{1}(N), \chi\right)$ is purely a technical convenience or whether it is natural in that, should it fail, there is a reason why $\lambda$ might not occur in the denominator of $L_{f}(k-1) /\left((2 \pi i)^{k-1} \Omega^{\kappa(k-1)}\right)$. In Section 8 we look into this and see how the failure of the condition might lead to nontrivial $\lambda$-torsion in $\amalg(k-1)$.

I am grateful to the referee for several helpful remarks, including the observation that more generally, if there is a newform $f \in S_{k}\left(\Gamma_{1}(N), \chi\right)$ with $f \equiv$ $E_{k}^{\chi, 1}\left(\bmod \lambda^{n}\right)$, then $\lambda^{n}$ divides both $\# H^{0}(\mathbb{Q}, A(k-1))$ and the denominator of $L_{f}(k-1) /\left((2 \pi i)^{k-1} \Omega^{\kappa(k-1)}\right)$, if $\lambda$ is not a congruence prime for $f$ in $S_{k}\left(\Gamma_{1}(N), \chi\right)$. The existence of such an $f$ may be deduced from $\lambda^{n} \mid L\left(1-k, \chi^{-1}\right)$ if $\lambda$ is not a congruence prime for $S_{k}\left(\Gamma_{1}(N)\right)$. For simplicity we only consider the case $n=1$.

\section{Eisenstein series and congruences with cusp forms}

Choose a weight $k \geq 3$, a level $N \geq 1$, and a Dirichlet character $\chi$ whose conductor divides $N$. Let $\psi$ and $\phi$ be primitive Dirichlet characters of conductors $u$ and $v$, respectively, with $\psi \phi=\chi, u v \mid N$, and $\chi(-1)=\kappa(k)$. Then there is an Eisenstein series $E_{k}^{\psi, \phi}$ belonging to $M_{k}\left(\Gamma_{1}(N), \chi\right)$. In fact, for all $\psi, \phi$ as above and positive $t$ such that $t \mid N /(u v)$, the $E_{k}^{\psi, \phi}(t \tau)$ form a basis for $M_{k}\left(\Gamma_{1}(N), \chi\right) / S_{k}\left(\Gamma_{1}(N), \chi\right)$. 
If $u v=N$ then $E_{k}^{\psi, \phi}$ is said to be new at $N$. If $k=2$, a slight modification is needed: for $N=1$ the (only) triple $\psi=\phi=\mathbf{1}, t=1$ must be excluded, and for $N>1$, $t \mid N$, one uses $E_{2}^{\mathbf{1 , 1}}(\tau)-t E_{2}^{\mathbf{1 , 1}}(t \tau)$ in place of $E_{2}^{\mathbf{1 , 1}}(t \tau)$.

At infinity, the $q$-expansion is

where

$$
E_{k}^{\psi, \phi}(\tau)=\delta(\psi) L\left(1-k, \psi^{-1} \phi\right)+2 \sum_{n=1}^{\infty} \sigma_{k-1}^{\psi, \phi}(n) q^{n},
$$

$$
\delta(\psi):=\left\{\begin{array}{ll}
1 & \text { if } \psi=\mathbf{1}, \\
0 & \text { otherwise, }
\end{array} \quad \text { and } \quad \sigma_{k-1}^{\psi, \phi}(n):=\sum_{m \mid n, m>0} \psi(n / m) \phi(m) m^{k-1} .\right.
$$

For all this, see [Diamond and Shurman 2005, Theorems 4.5.1 and 4.6.2]. Recall that $L\left(1-k, \psi^{-1} \phi\right) \in \mathbb{Q}\left(\psi^{-1} \phi\right)$, which is the extension of $\mathbb{Q}$ generated by the values of that character.

Proposition 2.1. Suppose that $E_{k}^{\psi, \phi}$ is new at level $N \geq 1$, with $k \geq 2$. Let $\lambda^{\prime} \nmid 6 N$ be a prime of $\mathbb{Z}[\psi, \phi]$ such that $\operatorname{ord}_{\lambda^{\prime}}\left(L\left(1-k, \psi^{-1} \phi\right)\right)>0$. Then there exists a normalised Hecke eigenform $f=\sum_{n=1}^{\infty} a_{n} q^{n} \in S_{k}\left(\Gamma_{1}(N), \chi\right)$ such that $a_{n} \equiv$ $\sigma_{k-1}^{\psi, \phi}(n)(\bmod \lambda)$ for all $n \geq 1$, where $\lambda \mid \lambda^{\prime}$ is a prime of the ring of integers of the extension of $\mathbb{Q}(\psi, \phi)$ generated by the $a_{n}$.

Proof. For any $\mathbb{Z}[1 / N]$-algebra $R$, let $M_{k}\left(\Gamma_{1}(N), R\right)$ be the $R$-module of Katz modular forms, and let $S_{k}\left(\Gamma_{1}(N), R\right)$ be the submodule of cusp forms. See [Edixhoven 1997, Section 1] for the definitions and basic properties. Consider $E_{k}^{\psi, \phi}$ as an element of $M_{k}\left(\Gamma_{1}(N), R\right)$, where $R=\mathbb{Z}\left[\zeta_{N}, \psi, \phi\right]_{\left(\lambda^{\prime \prime}\right)}, \lambda^{\prime \prime}$ is any prime divisor of $\lambda^{\prime}$ and $\zeta_{N}$ is a primitive $N^{\text {th }}$-root of unity. That we may do this follows from the $q$ expansion principle [Katz 1973,1.6] since $X_{1}(N)$ is connected and the coefficients in the $q$-expansion of $E_{k}^{\psi, \phi}$ at the cusp $\infty$ lie in $R$. According to [Faltings and Jordan 1995, Theorem 3.20], the constant term of $E_{k}^{\psi, \phi}$ at each (oriented) cusp is of the form $u L\left(1-k, \psi^{-1} \phi\right)$, with $u$ a unit in $\mathbb{Z}\left[1 /(2 N), \zeta_{N}, \psi, \phi\right]$. Therefore, since $\operatorname{ord}_{\lambda^{\prime}}\left(L\left(1-k, \psi^{-1} \phi\right)\right)>0$, we have $\overline{E_{k}^{\psi,}, \phi} \in S_{k}\left(\Gamma_{1}(N), \mathbb{F}_{\lambda^{\prime \prime}}\right)$, where $\overline{E^{\psi, \phi}}$ denotes the base-change of $E_{k}^{\psi, \phi}$ to $M_{k}\left(\Gamma_{1}(N), \mathbb{F}_{\lambda^{\prime \prime}}\right)$, and $\mathbb{F}_{\lambda^{\prime \prime}}$ is the residue field.

For each prime $p$ let $T_{p}$ be the Hecke operator for $\Gamma_{1}(N)$, and for $(d, N)=1$ let $\langle d\rangle$ be the diamond operator. Then by [Diamond and Shurman 2005, Proposition 5.2.3], $E_{k}^{\psi, \phi}$ is an eigenfunction for all the $T_{p}$ and $\langle d\rangle$, in fact,

$$
\begin{aligned}
T_{p} E_{k}^{\psi, \phi} & =\left(\psi(p)+\phi(p) p^{k-1}\right) E_{k}^{\psi, \phi} \\
\langle d\rangle E_{k}^{\psi, \phi} & =\chi(d) E_{k}^{\psi, \phi} .
\end{aligned}
$$

For $p \mid N$ this uses the fact that $E_{k}^{\psi, \phi}$ is new. The same equations hold for $\overline{E^{\psi, \phi}}$ in $S_{k}\left(\Gamma_{1}(N), \mathbb{F}_{\lambda^{\prime \prime}}\right)$. By [Edixhoven 1997, Lemma 1.9], the base-change map from $S_{k}\left(\Gamma_{1}(N), \overline{\mathbb{Z}_{\ell}}\right)$ to $S_{k}\left(\Gamma_{1}(N), \overline{\mathbb{F}_{\ell}}\right)$ is surjective, where $\lambda \mid \ell$. (Note that if $N \neq 1$ 
or $k \not \equiv 2(\bmod 12)$, we could allow $\ell=3$.) The existence of an eigenform $f \in$ $S_{k}\left(\Gamma_{1}(N)\right)$ with eigenvalues satisfying the desired congruences now follows easily (if not quite directly) from [Deligne and Serre 1974, Lemme 6.11]. That we may take $f \in S_{k}\left(\Gamma_{1}(N), \chi\right)$ is a consequence of Carayol's lemma [Edixhoven 1997, $1.10]$.

Note that, since the character $\chi$ has maximal conductor $N, f$ is a newform for $\Gamma_{1}(N)$. (Recall that $\psi \phi=\chi$, and we assumed that $E_{k}^{\psi, \phi}$ is new.)

\section{The Bloch-Kato conjecture}

For $k \geq 2, N \geq 1$ and a character $\chi$ whose conductor divides $N$, let $f=\sum_{n=1}^{\infty} a_{n} q^{n} \in$ $S_{k}\left(\Gamma_{1}(N), \chi\right)$ be a normalised newform. Attached to $f$ is its $L$-function $L_{f}(s)$, which is defined by the Dirichlet series $\sum_{n=1}^{\infty} a_{n} n^{-s}$ for $\Re(s)>(k+1) / 2$ but has an analytic continuation to the whole complex plane. Also attached to $f$ is a "premotivic structure" $M_{f}$ over $\mathbb{Q}$ with coefficients in $K$, the extension of $\mathbb{Q}(\chi)$ generated by the $a_{n}$. Thus there are 2-dimensional $K$-vector spaces $M_{f, B}$ and $M_{f, \mathrm{dR}}$ (the Betti and de Rham realisations) and, for each finite prime $\lambda$ of $O_{K}$, a 2-dimensional $K_{\lambda}$-vector space $M_{f, \lambda}$, the $\lambda$-adic realisation. These come with various structures and comparison isomorphisms, such as $M_{f, B} \otimes_{K} K_{\lambda} \simeq M_{f, \lambda}$. See [Diamond et al. 2004, 1.1.1] for the precise definition of a premotivic structure, and see [Diamond et al. 2004, 1.6.2] for the construction of $M_{f}$. The $\lambda$-adic realisation $M_{f, \lambda}$ comes with a continuous linear action of $\mathrm{Gal}(\overline{\mathbb{Q}} / \mathbb{Q})$. For each prime number $p$, the restriction to $\operatorname{Gal}\left(\overline{\mathbb{Q}}_{p} / \mathbb{Q}_{p}\right)$ may be used to define a local $L$-factor, and the Euler product is precisely $L_{f}(s)$ [Carayol 1986]. As the $L$-function attached to a premotivic structure, its orders of vanishing and leading terms at integer points may be interpreted via the Bloch-Kato conjecture.

On $M_{f, B}$ there is an action of $\operatorname{Gal}(\mathbb{C} / \mathbb{R})$, and the eigenspaces $M_{f, B}^{ \pm}$are 1dimensional. On $M_{f, \mathrm{dR}}$ there is a decreasing filtration, with $F^{j}$ a 1-dimensional space precisely for $1 \leq j \leq k-1$. The de Rham isomorphism $M_{f, B} \otimes_{K} \mathbb{C} \simeq$ $M_{f, \mathrm{dR}} \otimes_{K} \mathbb{C}$ induces isomorphisms between $M_{f, B}^{ \pm} \otimes \mathbb{C}$ and $\left(M_{f, \mathrm{dR}} / F\right) \otimes \mathbb{C}$, where $F:=F^{1}=\cdots=F^{k-1}$. Define $\Omega^{ \pm}$to be the determinants of these isomorphisms. These depend on the choice of $K$-bases for $M_{f, B}^{ \pm}$and $M_{f, \mathrm{dR}} / F$, so they should be viewed as elements of $\mathbb{C}^{\times} / K^{\times}$. For $1 \leq j \leq k-1$, the Tate-twisted premotivic structure $M_{f}(j)$ is critical (that is, the above map is an isomorphism, with $F=F^{j}$ ), and its Deligne period $c^{+}$(see [Deligne 1979]) is $(2 \pi i)^{j} \Omega^{\kappa(j)}$. Deligne's conjecture for $M_{f}(j)$, known in this case, asserts then that $L_{f}(j) /(2 \pi i)^{j} \Omega^{\kappa(j)}$ is an element of $K$.

If we choose $K$-bases for $M_{f, B}$ and $M_{f, \mathrm{dR}}$ to pin down $\Omega^{ \pm}$, then the BlochKato conjecture predicts the prime factorisation of the element $L_{f}(j) /(2 \pi i)^{j} \Omega^{\kappa(j)}$ of $K$. In fact, we shall choose an $O_{K}$-submodule $\mathfrak{M}_{f, B}$ that generates $M_{f, B}$ over 
$K$ but is not necessarily free, and likewise choose an $O_{K}[1 / S]$-submodule $\mathfrak{M}_{f, \mathrm{dR}}$ that generates $M_{f \text {,dR }}$ over $K$, where $S$ is the set of primes dividing $N k !$. We take these as in [Diamond et al. 2004, 1.6.2]. They are part of the " $S$-integral premotivic structure" associated to $f$. With these choices, it is still natural to talk of an element " $L_{f}(j) /(2 \pi i)^{j} \Omega^{\kappa(j)}$ " of the group of fractional ideals of $O_{K}[1 / S]$, and the BlochKato conjecture predicts its prime factorisation.

To define the various terms appearing in the conjecture, we shall need the elements $\mathfrak{M}_{f, \lambda}$ of the $S$-integral premotivic structure, for each prime $\lambda$ of $O_{K}$, and also the crystalline realisation $\mathfrak{M}_{f, \lambda \text {-crys }}$ for each $\lambda \notin S$. We choose these as in [Diamond et al. 2004, 1.6.2]. For each $\lambda, \mathfrak{M}_{f, \lambda}$ is a $\operatorname{Gal}(\overline{\mathbb{Q}} / \mathbb{Q})$-stable $O_{\lambda}$-lattice in $M_{f, \lambda}$. Let $A_{\lambda}:=M_{f, \lambda} / \mathfrak{M}_{f, \lambda}$. Define $\check{A}_{\lambda}:=\check{M}_{f, \lambda} / \check{\mathfrak{M}}_{f, \lambda}$, where $\check{M}_{f, \lambda}$ is the vector space dual to $M_{f, \lambda}$, where $\check{\mathfrak{M}}_{f, \lambda}$ is $O_{\lambda}$-lattice dual to $\mathfrak{M}_{f, \lambda}$, and where both are acted upon naturally by $\mathrm{Gal}(\overline{\mathbb{Q}} / \mathbb{Q})$. Let $A:=\oplus_{\lambda} A_{\lambda}$, et cetera.

Following [Bloch and Kato 1990, Section 3] for $p \neq \ell$ (including $p=\infty$ ), let

$$
H_{f}^{1}\left(\mathbb{Q}_{p}, M_{f, \lambda}(j)\right)=\operatorname{ker}\left(H^{1}\left(D_{p}, M_{f, \lambda}(j)\right) \rightarrow H^{1}\left(I_{p}, M_{f, \lambda}(j)\right)\right) .
$$

Here $D_{p}$ is a decomposition subgroup at a prime above $p, I_{p}$ is the inertia subgroup, and $M_{f, \lambda}(j)$ is a Tate twist of $M_{f, \lambda}$, et cetera. The cohomology is for continuous cocycles and coboundaries. For $p=\ell$, let

$$
H_{f}^{1}\left(\mathbb{Q}_{\ell}, M_{f, \lambda}(j)\right)=\operatorname{ker}\left(H^{1}\left(D_{\ell}, M_{f, \lambda}(j)\right) \rightarrow H^{1}\left(D_{\ell}, M_{f, \lambda}(j) \otimes_{\mathbb{Q}_{\ell}} B_{\text {crys }}\right)\right) .
$$

(See [Bloch and Kato 1990, Section 1] for the definition of Fontaine's ring $B_{\text {crys. }}$.) Let $H_{f}^{1}\left(\mathbb{Q}, M_{f, \lambda}(j)\right)$ be the subspace of those elements of $H^{1}\left(\mathbb{Q}, M_{f, \lambda}(j)\right)$ whose local restrictions lie in $H_{f}^{1}\left(\mathbb{Q}_{p}, M_{f, \lambda}(j)\right)$ for all primes $p$. There is a natural exact sequence

$$
0 \longrightarrow \mathfrak{M}_{f, \lambda}(j) \longrightarrow M_{f, \lambda}(j) \stackrel{\pi}{\longrightarrow} A_{\lambda}(j) \longrightarrow 0 .
$$

Define $H_{f}^{1}\left(\mathbb{Q}_{p}, A_{\lambda}(j)\right)=\pi_{*} H_{f}^{1}\left(\mathbb{Q}_{p}, M_{f, \lambda}(j)\right)$, and define the $\lambda$-Selmer group $H_{f}^{1}\left(\mathbb{Q}, A_{\lambda}(j)\right)$ to be the subgroup of elements of $H^{1}\left(\mathbb{Q}, A_{\lambda}(j)\right)$ whose local restrictions lie in $H_{f}^{1}\left(\mathbb{Q}_{p}, A_{\lambda}(j)\right)$ for all primes $p$. Note that the condition at $p=\infty$ is superfluous unless $\ell=2$. Define the Shafarevich-Tate group

$$
\amalg(j)=\bigoplus_{\lambda} \frac{H_{f}^{1}\left(\mathbb{Q}, A_{\lambda}(j)\right)}{\pi_{*} H_{f}^{1}\left(\mathbb{Q}, M_{f, \lambda}(j)\right)} .
$$

For a finite prime $p$, let $H_{f}^{1}\left(\mathbb{Q}_{p}, \mathfrak{M}_{f, \lambda}(j)\right)$ be the inverse image of $H_{f}^{1}\left(\mathbb{Q}_{p}, M_{f, \lambda}(j)\right)$ under the natural map. Suppose now that $p \neq \ell$. If $k$ is odd, suppose also that $j \neq(k-1) / 2$. Then $H^{0}\left(\mathbb{Q}_{p}, M_{f, \lambda}(j)\right)$ is trivial (because the eigenvalues of Frob $_{p}^{-1}$ 
acting on $M_{f, \lambda}$ are algebraic integers of absolute value $\left.p^{(k-1) / 2}\right)$. By inflationrestriction, $H_{f}^{1}\left(\mathbb{Q}_{p}, M_{f, \lambda}(j)\right) \simeq\left(M_{f, \lambda}(j)^{I_{p}}\right) /\left(1-\operatorname{Frob}_{p}\right)\left(M_{f, \lambda}(j)^{I_{p}}\right)$. This is trivial, since $H^{0}\left(\mathbb{Q}_{p}, M_{f, \lambda}(j)\right)$ is. Hence, using the exact sequence above, we see $H_{f}^{1}\left(\mathbb{Q}_{p}, \mathfrak{M}_{f, \lambda}(j)\right)$ is the torsion part of $H^{1}\left(\mathbb{Q}_{p}, \mathfrak{M}_{f, \lambda}(j)\right)$. Reusing the triviality of $H^{0}\left(\mathbb{Q}_{p}, M_{f, \lambda}(j)\right)$, we may identify $H_{f}^{1}\left(\mathbb{Q}_{p}, \mathfrak{M}_{f, \lambda}(j)\right)$ with $H^{0}\left(\mathbb{Q}_{p}, A_{\lambda}(j)\right)$.

This has an $O_{\lambda}$-submodule that is given by $\left(M_{f, \lambda}(j)^{I_{p}} / \mathfrak{M}_{f, \lambda}(j)^{I_{p}}\right)^{\text {Frob }_{p}=\text { id }}$ and whose "order" - that is, $\lambda$ raised to its length — is the $\lambda$-part of $P_{p}\left(p^{-j}\right)$, where $P_{p}\left(p^{-s}\right)=\operatorname{det}\left(1-\right.$ Frob $\left._{p}^{-1} p^{-s} \mid\left(M_{f, \lambda}\right)^{I_{p}}\right)$ is the Euler factor at $p$ in $L_{f}(s)$ (strictly speaking, its reciprocal). When $p \nmid N$, so that $M_{f, \lambda}(j)^{I_{p}}=M_{f, \lambda}(j)$ maps surjectively to $A_{\lambda}(j)$, the submodule is the whole of $H^{0}\left(\mathbb{Q}_{p}, A_{\lambda}(j)\right)$, but in general we define the $\lambda$-part of the Tamagawa factor $c_{p}(j)$ to be the index of the submodule.

It is also possible to define a $\lambda$-part of $c_{p}(j)$ for $\lambda \mid p$, using a measure of $H_{f}^{1}\left(\mathbb{Q}_{p}, \mathfrak{M}_{f, \lambda}(j)\right)$ arising from the Bloch-Kato exponential map. See [Bloch and Kato 1990] for details.

Conjecture 3.1. Suppose that $1 \leq j \leq k-1$. Then the Bloch-Kato conjecture predicts the following equality of fractional ideals of $O_{K}[1 / S]$ :

$$
\frac{L_{f}(j)}{(2 \pi i)^{j} \Omega^{\kappa(j)}}=\frac{\prod_{p \leq \infty} c_{p}(j) \# \amalg(j)}{\# H^{0}(\mathbb{Q}, A(j)) \# H^{0}(\mathbb{Q}, \check{A}(1-j))} .
$$

The Tamagawa factor $c_{\infty}(j)$ is at worst a power of 2 . We shall ignore \# $\amalg(j)$, except to note that it is integral. By [Dummigan et al. 2003, Lemmas 4.3 and 4.6], for $\lambda \nmid S$ the $\lambda$-part of $c_{p}(j)$ can only possibly be nontrivial if $p \mid N$. (The proof of [Lemma 4.6], that is, the case $\lambda \mid p$, uses $\mathbb{V}\left(\mathfrak{M}_{f, \lambda \text {-crys }}\right)=\mathfrak{M}_{f, \lambda}$, where $\mathbb{V}$ is the version of the Fontaine-Lafaille functor used in [Diamond et al. 2004].)

In Section 7 we shall show, under a certain condition, that for $f$ and $\lambda$ as in Proposition 2.1 with $\psi=\chi$ and $\phi=\mathbf{1}$ (and also $\lambda \nmid k$ !), we have

$$
\operatorname{ord}_{\lambda}\left(\frac{L_{f}(k-1)}{(2 \pi i)^{k-1} \Omega^{\kappa(k-1)}}\right)<0 .
$$

(The condition is that $\lambda$ is not a "congruence prime" for $f$ in $S_{k}\left(\Gamma_{1}, \chi\right)$.) Given that $\operatorname{ord}_{\lambda}(\# \amalg(k-1)) \geq 0$ and $\operatorname{ord}_{\lambda}\left(c_{p}(k-1)\right) \geq 0$ (for $p \nmid N$ it is actually 0$)$, the Bloch-Kato conjecture predicts that $H^{0}\left(\mathbb{Q}, A_{\lambda}(k-1)\right)$ or $H^{0}\left(\mathbb{Q}, \check{A}_{\lambda}(2-k)\right)$ must be nonzero. Now $\check{A}[\lambda](2-k)$ has composition factors $\mathbb{F}_{\lambda}(\chi)(2-k)$ and $\mathbb{F}_{\lambda}(1)-$ see the first paragraph of Section 4 - neither of which is trivial. Note that $\mathbb{F}_{\lambda}(\chi)$ denotes a 1-dimensional $\mathbb{F}_{\lambda}$-vector space on which $\mathrm{Gal}(\overline{\mathbb{Q}} / \mathbb{Q})$ acts via the reduction of the character $\chi$. The nontriviality of $\mathbb{F}_{\lambda}(\chi)(2-k)$ follows from $\ell>k$ and the fact that $\chi$ is unramified, but nontrivial if $k=2$. Hence $H^{0}\left(\mathbb{Q}, \check{A}_{\lambda}(2-k)\right)$ is zero. We shall confirm in the next section that $H^{0}\left(\mathbb{Q}, A_{\lambda}(k-1)\right)$ is nonzero.

In fact, we shall show also that $\operatorname{ord}_{\lambda}\left(c_{p}(k-1)\right)=0$ even for $p \mid N$, so that the contribution of $H^{0}\left(\mathbb{Q}, A_{\lambda}(k-1)\right)$ to the denominator is not cancelled by Tamagawa 
factors. This depends on $\chi$ having conductor exactly $N$, as will the proof that

$$
\operatorname{ord}_{\lambda}\left(\frac{L_{f}(k-1)}{(2 \pi i)^{k-1} \Omega^{\kappa(k-1)}}\right)<0 .
$$

We mention that the functional equation relates $L_{f}(s)$ to $L_{f_{\chi}^{-1}}(k-s)$. The form $f_{\chi^{-1}}$, the twist of $f$ by the character $\chi^{-1}$, lives in $S_{k}\left(\Gamma_{1}(N), \chi^{-1}\right)$, and it is congruent to $E_{k}^{\mathbf{1}, \chi^{-1}}(\bmod \lambda)$.

\section{Global torsion and Tamagawa factors}

Recall that we have chosen a weight $k \geq 2$, a level $N \geq 1$, a character $\chi$ of conductor precisely $N$ with $\chi(-1)=\kappa(k)$, and a cusp form $f \equiv E_{k}^{\chi, 1}(\bmod \lambda)$, where $\lambda \nmid$ $6 N k$ ! is a prime of $O_{K}$ (K being the extension of $\mathbb{Q}(\chi)$ generated by the Fourier coefficients $a_{n}$ of $f$ at $\left.\infty\right)$ such that $\operatorname{ord}_{\lambda}\left(L\left(1-k, \chi^{-1}\right)\right)>0$. For all primes $p \nmid N$, $a_{p} \equiv \chi(p)+p^{k-1}(\bmod \lambda)$. In fact this holds even for $p \mid N$, with $\chi(p)=0$ for such $p$. Since $a_{p}$ is the trace of $\operatorname{Frob}_{p}^{-1}$ on $M_{f, \lambda}$, it follows that $A[\lambda]$ (that is, the $\lambda$-torsion in $\left.A_{\lambda}\right)$ is reducible as an $\mathbb{F}_{\lambda}[\mathrm{Gal}(\overline{\mathbb{Q}} / \mathbb{Q})]$-module, with composition factors $\mathbb{F}_{\lambda}\left(\chi^{-1}\right)$ and $\mathbb{F}_{\lambda}(1-k)$. Here we identify $\chi$ with a character of $\operatorname{Gal}(\overline{\mathbb{Q}} / \mathbb{Q})$ via an Artin map that sends $p$ to Frob $_{p}$.

Theorem 4.1. In the situation of the preceding paragraph, $A[\lambda]$ has $\mathbb{F}_{\lambda}(1-k)$ as a submodule.

Corollary 4.2. $H^{0}\left(\mathbb{Q}, A_{\lambda}(k-1)\right)$ is nontrivial.

Before proving this we need a few preliminaries. Diamond, Flach and Guo, in [Diamond et al. 2004, 1.4.2], construct "premotivic structures" $M(N, \chi), M(N, \chi)_{c}$, and $M(N, \chi)$ ! for the space of modular forms of level $N$ and character $\chi$. Fixing choices of $N$ and $\chi$, we call these $M, M_{c}$ and $M_{!}$. (There is a map from $M_{c}$ to $M$ with image $M_{!}$.) Each has Betti, de Rham, and (for each prime $\lambda$ of $O_{K}$ ) $\lambda$-adic realisations, denoted $M_{B}, M_{\mathrm{dR}}, M_{\lambda}$, and so on. The Betti and de Rham realisations are $K$-vector spaces and the $\lambda$-adic realisations are $K_{\lambda}$-vector spaces. Here we choose $K$ as above, though the construction works for any number field containing $\mathbb{Q}(\chi)$. Temporarily $\lambda$ denotes any prime of $O_{K}$. There are various additional structures and comparison maps, discussed in detail in [Diamond et al. 2004]. For example, $M_{\lambda}$ supports a continuous representation of $\mathrm{Gal}(\overline{\mathbb{Q}} / \mathbb{Q})$. There are also $S$ integral premotivic structures $\mathfrak{M}, \mathfrak{M}_{c}$ and $\mathfrak{M}_{\text {! }}$, where $S$ is the set of primes dividing $N k$ !. These have realisations $\mathfrak{M}_{B}$ (an $O_{K}$-lattice in $M_{B}$ ), $\mathfrak{M}_{\mathrm{dR}}$ (an $O_{K}[1 / S]$-lattice in $\left.M_{\mathrm{dR}}\right)$, and $\mathfrak{M}_{\lambda}\left(\mathrm{a} \mathrm{Gal}(\overline{\mathbb{Q}} / \mathbb{Q})\right.$-stable $O_{\lambda}$-lattice in $M_{\lambda}$ ) for all primes $\lambda$, et cetera. There are canonical isomorphisms such as $\mathfrak{M}_{B} \otimes_{O_{K}} O_{\lambda} \simeq \mathfrak{M}_{\lambda}$. For $\lambda \notin S$ there is also a crystalline realisation $\mathfrak{M}_{\lambda \text {-crys }}$.

Let $\mathbb{T}^{\prime}$ be the ring generated over $O_{K}$ by all the Hecke operators $T_{n}$ acting on $M_{k}\left(\Gamma_{1}(N), \chi\right)$. There are compatible actions of $\mathbb{T}^{\prime}$ on all of the above, by 
[Diamond et al. 2004, Proposition 1.3]. Let $\Phi$ be the ideal of $\mathbb{T}^{\prime}$ generated by $T_{p}-\left(\chi(p)+p^{k-1}\right)$ for all primes $p$, and let $\mathfrak{m}$ be the maximal ideal generated by $\Phi$ and $\lambda$.

For $\lambda \notin S, \mathfrak{M}_{\lambda \text {-crys }}$ is a filtered $O_{\lambda}$ module with graded pieces of degrees 0 and $k-$ 1. There is a Hecke-equivariant isomorphism $\mathrm{Fil}^{k-1} \mathfrak{M}_{\lambda \text {-crys }} \simeq M_{k}\left(\Gamma_{1}(N), \chi, O_{\lambda}\right)$. It has an injective Frobenius endomorphism $\phi$ and is strongly divisible in the sense that $\mathfrak{M}_{\lambda \text {-crys }}=\phi \mathfrak{M}_{\lambda \text {-crys }}+\phi_{k-1}\left(\mathrm{Fil}^{k-1} \mathfrak{M}_{\lambda \text {-crys }}\right)$, where $\ell^{k-1} \phi_{k-1}: \mathrm{Fil}^{k-1} \mathfrak{M}_{\lambda \text {-crys }} \rightarrow$ $\mathfrak{M}_{\lambda \text {-crys }}$ is the restriction of $\phi$; see the end of [Diamond et al. 2004, 1.4.2]. Similar statements apply to $\mathfrak{M}_{c}$ and $\mathfrak{M}_{!}$when the submodule $M_{k}\left(\Gamma_{1}(N), \chi, O_{\lambda}\right)$ is replaced by $S_{k}\left(\Gamma_{1}(N), \chi, O_{\lambda}\right)$. When $\mathfrak{M}_{\lambda}, \mathfrak{M}_{c, \lambda}$, and $\mathfrak{M}_{!, \lambda}$ are viewed as $\mathbb{Z}_{\ell}$-modules with the $\operatorname{Gal}\left(\overline{\mathbb{Q}}_{\ell} / \mathbb{Q}_{\ell}\right)$-action, they may be identified respectively with $\mathbb{V}\left(\mathfrak{M}_{\lambda \text {-crys }}\right)$, $\mathbb{V}\left(\mathfrak{M}_{c, \lambda \text {-crys }}\right)$, and $\mathbb{V}\left(\mathfrak{M}_{!, \lambda \text {-crys }}\right)$, where $\mathbb{V}$ is the covariant version of Fontaine and Lafaille's functor used in [Diamond et al. 2004].

Lemma 4.3. Suppose that $\lambda \notin S, \lambda \nmid 6$, and $\operatorname{ord}_{\lambda}\left(L\left(1-k, \chi^{-1}\right)\right)>0$. The $\mathbb{F}_{\lambda}[\mathrm{Gal}(\overline{\mathbb{Q}} / \mathbb{Q})]$-module $\left(\mathfrak{M}_{!, \lambda} / \lambda \mathfrak{M}_{!, \lambda}\right)[\mathfrak{m}]$ has a submodule that becomes isomorphic to $\mathbb{F}_{\lambda}(1-k)$ upon restriction to $\mathrm{Gal}\left(\overline{\mathbb{Q}} / \mathbb{Q}\left(\zeta_{N}\right)\right)$, and it is the unique subquotient with this property.

Proof. This is based on the proof of [Faltings and Jordan 1995, Proposition 4.6]. The rank-one $O_{\lambda}$-submodule $\mathscr{E}$ of $M_{k}\left(\Gamma_{1}(N), \chi, O_{\lambda}\right)$ generated by the Eisenstein series $E_{k}^{\chi, 1}$ is the kernel of $\mathscr{I}$ on $\mathfrak{M}_{\lambda \text {-crys }}$, and so it is stable under $\phi_{k-1}$, since $\phi$ commutes with the Hecke operators. Since $\phi$ is injective and $\mathfrak{M}_{\lambda \text {-crys }}$ is strongly divisible, we must have $\phi_{k-1}(\mathscr{E})=\mathscr{E}$, so $\mathscr{E}$ is a strongly divisible filtered $\phi$-module. The functor $\mathbb{V}$ takes $\mathscr{E}$ to a rank-one $O_{\lambda}$-submodule $E$ of $\mathfrak{M}_{\lambda}$, which is stable under $\operatorname{Gal}\left(\overline{\mathbb{Q}}_{\ell} / \mathbb{Q}_{\ell}\right)$. In fact, since $\mathbb{V}$ respects Hecke operators, we have $E=\mathfrak{M}_{\lambda}[\mathscr{I}]$, and so it is stable under $\operatorname{Gal}(\overline{\mathbb{Q}} / \mathbb{Q})$. In fact, $E \simeq O_{\lambda}(1-k)$ as a $\operatorname{Gal}\left(\overline{\mathbb{Q}} / \mathbb{Q}\left(\zeta_{N}\right)\right)$-module since $M_{!, \lambda}[\Phi]=0$, and by [Scholl 1990, 1.2.0] the cokernel of the inclusion of $M_{!, \lambda}$ in $M_{\lambda}$ becomes, upon restriction to $\operatorname{Gal}\left(\overline{\mathbb{Q}} / \mathbb{Q}\left(\zeta_{N}\right)\right)$, isomorphic to a direct sum of copies of $K_{\lambda}(1-k)$.

Let $M_{k}=M_{k}\left(\Gamma_{1}(N), \chi, O_{\lambda}\right)$ and $S_{k}=S_{k}\left(\Gamma_{1}(N), \chi, O_{\lambda}\right)$. The image of $\mathscr{E}$ in $M_{k} / \lambda M_{k}$ actually lies in $S_{k} / \lambda S_{k}$, as noted in the proof of Proposition 2.1. This gives a $\phi_{k-1}$-stable, one-dimensional $\mathbb{F}_{\lambda}$-subspace $\overline{\mathscr{E}}$ of the finite-length filtered $O_{\lambda}$-module $\mathfrak{M}_{!, \lambda \text {-crys }} / \lambda \mathfrak{M}_{!, \lambda \text {-crys. }}$ This subspace lies inside $\mathrm{Fil}^{k-1}$, and since $\mathscr{E}$ is killed by $\mathscr{I}$, we have $\overline{\mathscr{E}} \subset\left(\mathfrak{M}_{!, \lambda \text {-crys }} / \lambda \mathfrak{M}_{!, \lambda \text {-crys }}\right)[\mathfrak{m}]$.

We may apply a finite-length version of the functor $\mathbb{V}$ (see [Diamond et al. $2004,1.1 .2])$ to get a one-dimensional subspace $W$ of $\left(\mathfrak{M}_{!, \lambda} / \lambda \mathfrak{M}_{!, \lambda}\right)[\mathfrak{m}]$. Inside $\left(\mathfrak{M}_{\lambda} / \lambda \mathfrak{M}_{\lambda}\right)[\mathfrak{m}], W$ is just the reduction of $E$ and so is isomorphic to $\mathbb{F}_{\lambda}(1-k)$ as a module for $\operatorname{Gal}\left(\overline{\mathbb{Q}} / \mathbb{Q}\left(\zeta_{N}\right)\right)$. Let $L$ be the finite unramified extension of $\mathbb{Q}_{\ell}$ corresponding to $\mathbb{Q}\left(\zeta_{N}\right)$, and let $\psi$ be any $\left(O_{\lambda}\right.$-valued) character of $\operatorname{Gal}\left(L / \mathbb{Q}_{\ell}\right)$. 
By the $q$-expansion principle, $\operatorname{dim}_{\mathbb{F}_{\lambda}}\left(S_{k}\left(\Gamma_{1}(N), \chi, \mathbb{F}_{\lambda}\right)[\mathfrak{m}]\right)=1$, and hence

$$
\mathrm{Fil}^{k-1}\left(\left(\left(\mathfrak{M}_{!, \lambda \text {-crys }} / \lambda \mathfrak{M}_{!, \lambda \text {-crys }}\right)[\mathfrak{m}]\right) / \overline{\mathscr{E}}\right)=0 .
$$

But

$$
\left.\mathbb{V}\left(\left(\mathfrak{M}_{!, \lambda \text {-crys }} / \lambda \mathfrak{M}_{!, \lambda \text {-crys }}\right)[\mathfrak{m}]\right) / \overline{\mathscr{E}}\right)=\left(\left(\mathfrak{M}_{!, \lambda} / \lambda \mathfrak{M}_{!, \lambda}\right)[\mathfrak{m}]\right) / W
$$

and the filtered module $\mathbb{F}_{\lambda}\{\psi\}\{1-k\}$ such that $\mathbb{V}\left(\mathbb{F}_{\lambda}\{\psi\}\{1-k\}\right)=\mathbb{F}_{\lambda}(\psi)(1-k)$ has $\mathrm{Fil}^{k-1} \mathbb{F}_{\lambda}\{\psi\}\{1-k\}=\mathbb{F}_{\lambda}\{\psi\}\{1-k\}$, and so $\left(\mathfrak{M}_{!, \lambda} / \lambda \mathfrak{M}_{!, \lambda}\right)[\mathfrak{m}]$ cannot have any more composition factors isomorphic to $\mathbb{F}_{\lambda}(1-k)$ upon restriction to $\operatorname{Gal}\left(\overline{\mathbb{Q}}_{\ell} / L\right)$.

Proof of Theorem 4.1. By construction, $\mathfrak{M}_{f, \lambda}$ is a submodule of $\mathfrak{M}_{!, \lambda}$, and hence $A[\lambda]$ is a submodule of $\left(\mathfrak{M}_{!, \lambda} / \lambda \mathfrak{M}_{!, \lambda}\right)[\mathfrak{m}]$. In the latter, as above, $\mathbb{F}_{\lambda}(1-k)$ has multiplicity at most one and appears as a submodule, if at all. It remains to observe that the subquotients of $A[\lambda]$ are $\mathbb{F}_{\lambda}\left(\chi^{-1}\right)$ and $\mathbb{F}_{\lambda}(1-k)$; so the latter must be a submodule. Note that since $\chi$ is unramified at $\ell$ and $k<\ell$, these factors remain distinct upon restriction to $\operatorname{Gal}\left(\overline{\mathbb{Q}}_{\ell} / L\right)$, the latter being ramified.

Write $\chi=\prod_{p \mid N} \chi_{p}$, where the conductor of $\chi_{p}$ is the power of $p$ in $N$.

Proposition 4.4. In the same situation as above, $\operatorname{ord}_{\lambda}\left(c_{p}(j)\right)=0$ for any integer $j$ and any prime $p \mid N$ such that the order of $\chi_{p}$ is not a power of $\ell$ (for example, if $\ell \nmid p-1)$.

Proof. Since $a_{p} \equiv \chi(p)+p^{k-1}=p^{k-1} \not \equiv 0(\bmod \lambda)$, the Euler factor $\left(1-a_{p} p^{-s}\right)$ must have degree 1 . Recalling that this Euler factor is the reciprocal of

$$
\operatorname{det}\left(1-\operatorname{Frob}_{p}^{-1} p^{-s} \mid\left(M_{f, \lambda}\right)^{I_{p}}\right),
$$

we see that $\operatorname{dim}\left(M_{f, \lambda}\right)^{I_{p}}=1$. Now $\operatorname{ord}_{\lambda}\left(c_{p}(j)\right)$ could be nonzero only if the map from $\left(M_{f, \lambda}\right)^{I_{p}}$ to $\left(A_{\lambda}\right)^{I_{p}}$ is not surjective. This would force $\operatorname{dim} A[\lambda]^{I_{p}}>1$, so $A[\lambda]^{I_{p}}=A[\lambda]$. This cannot be the case, since the composition factors of $A[\lambda]$ are $\mathbb{F}_{\lambda}(1-k)$ and $\mathbb{F}_{\lambda}\left(\chi^{-1}\right)$, and $\chi$, having exact conductor $N$, is ramified at $p$. The condition on the order of $\chi_{p}$ ensures that the reduction $(\bmod \lambda)$ of $\chi$ (which we also call $\chi$, by abuse of notation) is still ramified at $p$.

\section{The Hecke action on boundary symbols}

Let $R$ be a commutative ring in which 6 is invertible. Let $A$ be a right $R[\Sigma]$-module, where $\Sigma=M_{2}(\mathbb{Z}) \cap \mathrm{GL}_{2}(\mathbb{Q})$. Let $\mathscr{D}$ be the group of divisors supported on $\mathbb{P}^{1}(\mathbb{Q})$, with $\mathscr{D}_{0}$ the subgroup of divisors of degree zero. There is a natural left action of $\mathrm{GL}_{2}(\mathbb{Q})$ on $\mathscr{D}$. If $\Gamma$ is any congruence subgroup of $\operatorname{SL}_{2}(\mathbb{Z})$, let $\operatorname{Symb}_{\Gamma}(A)$ (the group of $A$-valued modular symbols for $\Gamma$ ) be the set of homomorphisms $\Phi: \mathscr{D}_{0} \rightarrow$ $A$ such that $\Phi \mid g=\Phi$ for all $g \in \Gamma$, where $(\Phi \mid g)(D):=(\Phi(g D)) \mid g$. Replacing $\mathscr{D}_{0}$ by $\mathscr{D}$, we likewise define $\operatorname{Bound}_{\Gamma}(A)$, the group of $A$-valued boundary symbols for 
$\Gamma$. Restriction from $\mathscr{D}$ to $\mathscr{D}_{0}$ provides a natural homomorphism from $\operatorname{Bound}_{\Gamma}(A)$ to $\operatorname{Symb}_{\Gamma}(A)$. A useful reference for modular symbols is [Greenberg and Stevens 1993, Section 4].

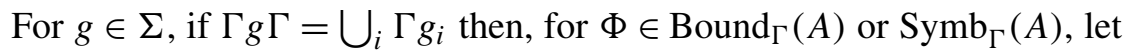

$$
\Phi\left|T(g):=\sum_{i} \Phi\right| g_{i}
$$

When $g=\left[\begin{array}{ll}1 & 0 \\ 0 & p\end{array}\right]$, we abbreviate $T(g)$ to $T(p)$.

From now on, we fix a choice of weight $k \geq 2$ and level $N$, and let $\Gamma=\Gamma_{1}(N)$. We let $A=\operatorname{Sym}^{k-2} R^{2}$ be the module of polynomials of degree $k-2$ over $R$ in variables $X$ and $Y$. The right $\Sigma$-action is defined by $(F \mid g)(X, Y)=F\left((X, Y) g^{*}\right)$, where for

$$
g=\left[\begin{array}{ll}
a & b \\
c & d
\end{array}\right], \quad \text { we define } \quad g^{*}:=\left[\begin{array}{rr}
d & -b \\
-c & a
\end{array}\right]
$$

Sometimes we may write $\operatorname{Symb}_{k}\left(\Gamma_{1}(N), R\right)$ instead of $\operatorname{Symb}_{\Gamma_{1}(N)}\left(\operatorname{Sym}^{k-2} R^{2}\right)$. If $\psi:(\mathbb{Z} / N \mathbb{Z})^{\times} \rightarrow R^{\times}$is a character, we may view $\psi$ as a character of $\Gamma_{0}(N)$ in the usual way: $\psi\left(\left[\begin{array}{ll}a & b \\ c & d\end{array}\right]\right)=\psi(d)$. Then let $\operatorname{Symb}_{k}\left(\Gamma_{1}(N), \psi, R\right):=\{\Phi \in$ $\operatorname{Symb}_{k}\left(\Gamma_{1}(N), R\right): \Phi \mid g=\psi(g) \Phi$ for all $\left.g \in \Gamma_{0}(N)\right\}$. Likewise for Bound. By [Greenberg and Stevens 1993, Theorem 4.3], $\operatorname{Bound}_{k}\left(\Gamma_{1}(N), \psi, R\right)$ may be viewed as a subgroup of $\operatorname{Symb}_{k}\left(\Gamma_{1}(N), \psi, R\right)$. In fact, using a theorem of Ash and Stevens (see Section 6 below) to identify $\operatorname{Symb}_{k}\left(\Gamma_{1}(N), \psi, R\right)$ with a certain compactly supported cohomology group, we see $\operatorname{Bound}_{k}\left(\Gamma_{1}(N), \psi, R\right)$ is the kernel of the projection onto parabolic cohomology.

Proposition 5.1. Let $\chi$ be an $R$-valued character of conductor $N$. There is an element $\Phi_{\chi}$ of $\operatorname{Bound}_{k}\left(\Gamma_{1}(N), \chi, R\right)$ that is supported on the $\Gamma_{0}(N)$-orbit of $\infty$ and is such that $\Phi_{\chi}(\infty)=X^{k-2}$. For all primes $p$, it satisfies

$$
\Phi_{\chi} \mid T(p)=\left(p^{k-1}+\chi(p)\right) \Phi_{\chi} .
$$

It spans the submodule of $\operatorname{Bound}_{k}\left(\Gamma_{1}(N), \chi, R\right)$ comprising all $\Phi$ on which the $T(p)$ act in this manner.

Proof. The stabiliser of $\infty$ in $\Gamma_{1}(N)$ is the subgroup generated by $\left[\begin{array}{ll}1 & 1 \\ 0 & 1\end{array}\right]$. The submodule of $\mathrm{Sym}^{k-2} R^{2}$ fixed by this subgroup is spanned by $X^{k-2}$. Therefore, up to a scalar, we are forced to choose $\Phi_{\chi}(\infty)=X^{k-2}$. Now the values of $\Phi_{\chi}$ on the $\Gamma_{0}(N)$-orbit of $\infty$ are determined by $\Phi_{\chi} \mid g=\chi(g) \Phi_{\chi}$, so that $\Phi_{\chi}(g \infty)=$ $\chi(g) \Phi_{\chi}(\infty) \mid g^{-1}$ for all $g \in \Gamma_{0}(N)$. We complete the definition of $\Phi_{\chi}$ by decreeing that it take the value 0 outside the $\Gamma_{0}(N)$-orbit of $\infty$. 
Next we calculate the action of $T(p)$. According to [Diamond and Shurman 2005, Proposition 5.2.1], if $p \nmid N$ then

$$
\Phi_{\chi}\left|T(p)=\sum_{j=0}^{p-1} \Phi_{\chi}\right|\left[\begin{array}{ll}
1 & j \\
0 & p
\end{array}\right]+\Phi_{\chi} \mid\left[\begin{array}{ll}
m & n \\
N & p
\end{array}\right]\left[\begin{array}{ll}
p & 0 \\
0 & 1
\end{array}\right],
$$

where $m p-n N=1$. In the sum from 0 to $p-1$, each $g_{i}$ fixes $\infty$, so each of the $p$ terms, evaluated at $\infty$, is $(p X)^{k-2}$. If $g=\left[\begin{array}{ll}m & n \\ N & p\end{array}\right]$ then $g \in \Gamma_{0}(N)$, and so $\Phi_{\chi} \mid g=\chi(p) \Phi_{\chi}$; so $\left(\Phi_{\chi} \mid g\right)(\infty)=\chi(p) X^{k-2}$. Now $\left[\begin{array}{ll}p & 0 \\ 0 & 1\end{array}\right]$ fixes both $\infty$ and $X^{k-2}$. So we find that

$$
\left(\Phi_{\chi} \mid T(p)\right)(\infty)=\left(p^{k-1}+\chi(p)\right) \Phi_{\chi}(\infty) .
$$

The fact that $T(p)$ commutes with the diamond operators $\langle d\rangle$ (see the calculation in [Diamond and Shurman 2005, p. 169]) allows us to extend this from $\infty$ to the whole $\Gamma_{0}(N)$-orbit of $\infty$.

It remains to sketch a proof of the uniqueness property of $\Phi_{\chi}$. If $\psi$ and $\phi$ are primitive Dirichlet characters of conductors $u$ and $v$, respectively, with $\psi \phi=\chi$ and $u v=N$, then we may apply the above construction to $\psi \phi^{-1}$ and then apply a twisting operator $\Phi\left|R_{\phi}=\sum_{a=0}^{v-1} \phi(a) \Phi\right|\left[\begin{array}{ll}1 & a \\ 0 & v\end{array}\right]$; see [Greenberg and Stevens 1993, 4.10]. Thus we get some $\Phi \in \operatorname{Bound}_{k}\left(\Gamma_{1}(N), \chi, R\right)$ such that, for all primes $p$, $\Phi \mid T(p)=\left(\phi(p) p^{k-1}+\psi(p)\right) \Phi$. If there were a $\Phi \in \operatorname{Bound}_{k}\left(\Gamma_{1}(N), \chi, R\right)$ that satisfies $\Phi \mid T(p)=\left(p^{k-1}+\chi(p)\right) \Phi$ for all primes $p$ and that is not a multiple of $\Phi_{\chi}$, then, together with what we have constructed, $\Phi$ would span over $\mathbb{C}$ a space of dimension greater than that of $M_{k}\left(\Gamma_{1}(N), \chi\right) / S_{k}\left(\Gamma_{1}(N), \chi\right)$; recall the first paragraph of Section 2. This would contradict that $\operatorname{Bound}_{k}\left(\Gamma_{1}(N), \chi, \mathbb{C}\right)$ has the same dimension as $M_{k}\left(\Gamma_{1}(N), \chi\right) / S_{k}\left(\Gamma_{1}(N), \chi\right)$. (This fact follows from comparing the dimensions of the graded pieces of $M_{c, \mathrm{dR}}$ and $M_{!, \mathrm{dR}}$.)

The spaces $\operatorname{Symb}_{k}\left(\Gamma_{1}(N), \chi, R\right)$ and $\operatorname{Bound}_{k}\left(\Gamma_{1}(N), \chi, R\right)$ are broken into \pm eigenspaces by the involution $\iota=\left[\begin{array}{rr}1 & 0 \\ 0 & -1\end{array}\right]$. It is easy to check that $\Phi_{\chi}$ is in the $\kappa(k-2)=\kappa(k)$-eigenspace, that is, $\Phi_{\chi} \mid \iota=\kappa(k) \Phi_{\chi}$.

\section{A congruence of modular symbols}

Let $R$ be a commutative ring in which 6 is invertible, $A$ be a right $R[\Sigma]$-module, and $\Gamma$ be any congruence subgroup of $\mathrm{SL}_{2}(\mathbb{Z})$. Then by a theorem of Ash and Stevens [1986] [Greenberg and Stevens 1993, Theorem 4.2], there is a natural isomorphism

$$
\operatorname{Symb}_{\Gamma}(A) \simeq H_{c}^{1}(\Gamma \backslash \mathfrak{H}, A) .
$$

Letting $\Gamma=\Gamma(N)$ and $A=\operatorname{Sym}^{k-2} R^{2}$ with $R=O_{\lambda}$, then taking the part on which $\Gamma_{0}(N)$ acts via $\chi$, we get $\operatorname{Symb}_{k}\left(\Gamma_{1}(N), \chi, O_{\lambda}\right)$ from the left, while from the right, 
following the construction in [Diamond et al. 2004, 1.2-1.4], we get $\mathfrak{M}_{c, \lambda}$. In other words:

Lemma 6.1. $\operatorname{Symb}_{k}\left(\Gamma_{1}(N), \chi, O_{\lambda}\right) \simeq \mathfrak{M}_{c, \lambda}$.

The action of $\iota$ on the left matches the action of $\operatorname{Gal}(\mathbb{C} / \mathbb{R})$ on the right.

Though it is not necessary for the truth of the lemma, we now put ourselves in the situation described in the first paragraph of Section 4. Recall that $\mathbb{T}^{\prime}$ is the ring generated over $O_{K}$ by all the Hecke operators $T_{n}$ acting on $M_{k}\left(\Gamma_{1}(N), \chi\right)$, and $\mathscr{I}$ is the ideal of $\mathbb{T}^{\prime}$ generated by $T_{p}-\left(\chi(p)+p^{k-1}\right)$ for all primes $p$. Let $I_{f}$ be the ideal of $\mathbb{T}^{\prime}$ generated by $T_{p}-a_{p}$ for all primes $p$. Define $\Phi_{f}$ to be any generator for the free rank-1 $O_{\lambda}$-module $\operatorname{Symb}_{k}\left(\Gamma_{1}(N), \chi, O_{\lambda}\right)^{\kappa(k)}\left[\mathscr{I}_{f}\right]$. Let $\Phi_{\chi}$ be as in Proposition 5.1. We say that $\lambda$ is a congruence prime for $f$ in $S_{k}\left(\Gamma_{1}(N), \chi\right)$ if there exists $g \in S_{k}\left(\Gamma_{1}(N), \chi, O_{K^{\prime}}\right)$ that is orthogonal to $f$ (with $K^{\prime}$ some sufficiently large finite extension of $K)$ such that $f \equiv g\left(\bmod \lambda^{\prime}\right)\left(\right.$ where $\left.\lambda^{\prime} \mid \lambda\right)$. By applying the eigenspace-killing procedure described in the proof below, one may assume that $g$ is an eigenvector for $\mathbb{T}^{\prime}$.

Lemma 6.2. Suppose that $\lambda$ is not a congruence prime for $f$ in $S_{k}\left(\Gamma_{1}(N), \chi\right)$. We may choose $\Phi_{f}$ in such a way that $\Phi_{f}-\Phi_{\chi} \in \lambda \operatorname{Symb}_{k}\left(\Gamma_{1}(N), \chi, O_{\lambda}\right)$.

Proof. Define

$$
\begin{aligned}
M_{f, E} & :=M_{\lambda}[\mathscr{I}] \oplus M_{\lambda}\left[\mathscr{I}_{f}\right], & \mathfrak{M}_{f, E} & :=M_{f, E} \cap \mathfrak{M}_{\lambda} \\
M_{c, f, E} & :=M_{c, \lambda}[\mathscr{I}] \oplus M_{c, \lambda}\left[\mathscr{I}_{f}\right], & \mathfrak{M}_{c, f, E} & :=M_{c, f, E} \cap \mathfrak{M}_{c, \lambda} .
\end{aligned}
$$

Since $\ell>k-2$, the duality morphisms of [Diamond et al. 2004, 1.5] induce perfect $O_{\lambda}$-valued pairings between $\mathfrak{M}_{c, \lambda}$ and $\mathfrak{M}_{\lambda}$, and between $\mathfrak{M}_{c, \lambda}^{\kappa(k)}$ and $\mathfrak{M}_{\lambda}^{\kappa(k-1)}$. Restriction gives a pairing between $\mathfrak{M}_{c, f, E}^{\kappa(k)}$ and $\mathfrak{M}_{f, E}^{\kappa(k-1)}$. Let

$$
\begin{gathered}
v_{f} \text { be a generator for } M_{\lambda}^{\kappa(k-1)}\left[\mathscr{I}_{f}\right] \cap \mathfrak{M}_{f, E}^{\kappa(k-1)} ; \\
v_{E} \text { be a generator for } M_{\lambda}^{\kappa(k-1)}[\mathscr{g}] \cap \mathfrak{M}_{f, E}^{\kappa(k-1)} ; \\
w_{f} \text { be a generator for } M_{c, \lambda}^{\kappa(k)}\left[\mathscr{I}_{f}\right] \cap \mathfrak{M}_{f, E}^{\kappa(k)} ; \\
w_{E} \text { be a generator for } M_{c, \lambda}^{\kappa(k)}[\mathscr{I}] \cap \mathfrak{M}_{f, E}^{\kappa(k)} .
\end{gathered}
$$

In fact we choose $w_{f}$ and $w_{E}$ to be the images of $\Phi_{f}$ and $\Phi_{\chi}$ under the isomorphism of Lemma 6.1. We wish to show that $w_{f}-w_{E} \in \lambda \mathfrak{M}_{c, f, E}^{\kappa(k)}$. Complex conjugation in $\operatorname{Gal}(\overline{\mathbb{Q}} / \mathbb{Q})$ acts on $\mathbb{F}_{\lambda}(1-k)$ as $\kappa(k-1)$ but on $\mathbb{F}_{\lambda}\left(\chi^{-1}\right)$ as $\kappa(k)$. Therefore it must be the former that is spanned by the image of $v_{f}$ in $\mathfrak{M}_{\lambda} / \lambda \mathfrak{M}_{f, \lambda}$. Applying Lemma 4.3 and choosing scalar multiples appropriately, we find without losing generality that $v_{f}-v_{E} \in \lambda \mathfrak{M}_{f, E}^{k(k-1)}$. Let $r$ be the largest integer such that $v_{f}-v_{E} \in \lambda^{r} \mathfrak{M}_{f, E}^{k(k-1)}$. Since the Hecke operators $T_{p}$ (for $p \nmid N$ ) are self-adjoint 
for the pairing, we have $\left\langle w_{f}, v_{E}\right\rangle=\left\langle w_{E}, v_{f}\right\rangle=0$. Now let $\lambda$ denote also some uniformiser in $O_{\lambda}$. If $v_{f}-v_{E}=\lambda^{r} v$, then we have

$$
\left\langle w_{f}, v_{f}\right\rangle=\lambda^{r}\left\langle w_{f}, v\right\rangle \quad \text { and } \quad\left\langle w_{E}, v_{E}\right\rangle=-\lambda^{r}\left\langle w_{E}, v\right\rangle .
$$

If $w_{f}$ and $w_{E}$ do not span $\mathfrak{M}_{c, f, E}^{\kappa(k)}$, then we may choose them in such a way that $w_{f}-w_{E} \in \lambda \mathfrak{M}_{c, f, E}^{\kappa(k)}$, as required. So suppose that $w_{f}$ and $w_{E}$ do span $\left(\mathfrak{M}_{c, f, E}\right)^{\kappa(k)}$.

By perfectness of the pairing between $\mathfrak{M}_{c, \lambda}^{k(k)}$ and $\mathfrak{M}_{\lambda}^{\kappa(k-1)}$, there exists some $g \in \mathfrak{M}_{\lambda}^{\kappa(k-1)}$ such that

$$
\left\langle w_{f}, g\right\rangle=1 \quad \text { and } \quad\left\langle w_{E}, g\right\rangle=0 .
$$

Then $g=\left(v_{f}-h\right) / \lambda^{r}$ for some $h \in \mathfrak{M}_{\lambda}^{\kappa(k-1)}$, that is, $h=v_{f}-\lambda^{r} g$. There is a decomposition of $M_{\lambda}^{\kappa(k-1)} \otimes K_{\lambda^{\prime}}^{\prime}$, when $K_{\lambda^{\prime}}^{\prime}$ is some sufficiently large finite extension of $K_{\lambda}$, into one-dimensional $\mathbb{T}^{\prime}$-eigenspaces, all "new" because $\chi$ has conductor $N$. This parallels the decomposition of $M_{k}\left(\Gamma_{1}(N), \chi\right)$. The element $h$ is a linear combination of eigenvectors, with $v_{E}$ and $v_{f}$ excluded, since by design $\left\langle h, w_{f}\right\rangle=\left\langle h, w_{E}\right\rangle=0$. If there is a system of eigenvalues $\left\{b_{p}\right\}$ such that $a_{p} \not \equiv b_{p}\left(\bmod \lambda^{\prime}\right)$ for some $p$, then by applying $\left(T_{p}-b_{p}\right) /\left(a_{p}-b_{p}\right)$, we can kill the eigenspace corresponding to $\left\{b_{p}\right\}$ in the expression $g=\left(v_{f}-h\right) / \lambda^{r}$. Since $h$ is necessarily nontrivial, there must be a system of eigenvalues $\left\{b_{p}\right\}$ (corresponding to some cusp form in $S_{k}\left(\Gamma_{1}(N), \chi\right)$ different from $\left.f\right)$ such that $a_{p} \equiv b_{p}\left(\bmod \lambda^{\prime}\right)$ for every $p$. In other words, $\lambda$ is a congruence prime for $f$ in $S_{k}\left(\Gamma_{1}(N), \chi\right)$.

\section{The denominator of the $L$-value}

Throughout this section, we are in the situation described in the first paragraph of Section 4. We need to consider the period $\Omega^{\kappa(k-1)}$. Since we are looking just at the $\lambda$-part of the Bloch-Kato conjecture, this period matters only up to a unit in $O_{(\lambda)}$, the localisation at $\lambda$ of $O_{K}$. Recall that in Section $3, \Omega^{ \pm}$were defined as determinants of isomorphisms from $M_{f, B}^{ \pm} \otimes \mathbb{C}$ to $\left(M_{f, \mathrm{dR}} / F\right) \otimes \mathbb{C}$, calculated with respect to bases arising from $\mathfrak{M}_{f, B}$ and $\mathfrak{M}_{f, \mathrm{dR}}$. (We can choose bases for $\mathfrak{M}_{f, B} \otimes O_{(\lambda)}$ and $\mathfrak{M}_{f, \mathrm{dR}} \otimes O_{(\lambda)}$.) Let $\omega^{ \pm}$be the determinants of isomorphisms going the other way, that is, from $F \otimes \mathbb{C}$ to $M_{f, B}^{ \pm} \otimes \mathbb{C}$, that arise from the inverse of the comparison isomorphism $I: M_{f, B} \otimes \mathbb{C} \rightarrow M_{f, \mathrm{dR}} \otimes \mathbb{C}$, also calculated with respect to bases coming from $\mathfrak{M}_{f, B}$ and $\mathfrak{M}_{f, \mathrm{dR}}$.

Lemma 7.1. Up to a unit in $O_{(\lambda)}$,

$$
\omega^{ \pm}=(2 \pi i)^{k-1} \Omega^{\mp} .
$$

Proof. Applying [Deligne 1979, Lemma 5.1.6], we find $\omega^{ \pm}=\Omega^{\mp} / \operatorname{det} I$, where the determinant is calculated using bases coming from $\mathfrak{M}_{f, B}$ and $\mathfrak{M}_{f, \mathrm{dR}}$. Now $\operatorname{det}\left(\mathfrak{M}_{f, B}\right)=\eta_{f} \mathfrak{M}_{\chi}(1-k)_{B}$ and $\operatorname{det}\left(\mathfrak{M}_{f, \mathrm{dR}}\right)=\eta_{f} \mathfrak{M}_{\chi}(1-k)_{\mathrm{dR}}$, in the notation of 
[Diamond et al. 2004, 1.7.3] $\left(\eta_{f}\right.$ is a certain fractional ideal of $K$ and $M_{\chi}(1-k)$ is a Tate-twist of a Dirichlet motive). We may realise det $I$ as the period of $M_{\chi}(1-$ $k$ ) with respect to the integral structure $\mathfrak{M}_{\chi}(1-k)$, namely $G(\chi)(2 \pi i)^{1-k}$; see [Diamond et al. 2004, 1.1.3]). The Gauss sum $G(\chi)$ is coprime to $\lambda$.

Recall from Section 4 the premotivic structures $M_{c}, M$, and $M_{\text {! }}$ associated with $M_{k}\left(\Gamma_{1}(N), \chi\right)$, and recall that $M_{\text {! }}$ is the image of $M_{c}$ in $M$. The premotivic structure $M_{f}$ attached to $f$ is $M_{!}\left[\mathscr{I}_{f}\right]$. Likewise $\mathfrak{M}_{f}=\mathfrak{M}_{!}\left[\mathscr{I}_{f}\right]$. There are natural identifications of $\mathrm{Fil}^{k-1} M_{c, \mathrm{dR}}$ and Fil ${ }^{k-1} M_{!, \mathrm{dR}}$ with $S_{k}\left(\Gamma_{1}(N), \chi, K\right)$, such that $\mathrm{Fil}^{k-1} \mathfrak{M}_{f, \mathrm{dR}}=O_{K}[1 / S] f$; see [Diamond et al. 2004, 1.4.2 and 1.6.2].

To $f$ we associate a modular symbol $\psi_{f} \in \operatorname{Symb}_{k}\left(\Gamma_{1}(N), \chi, \mathbb{C}\right)$ defined by

$$
\psi_{f}(\{b\}-\{a\})=(2 \pi i)^{k-1} \int_{a}^{b} f(\tau)(\tau X+Y)^{k-2} d \tau .
$$

Via the Ash-Stevens isomorphism we may view it as an element of $M_{c, B} \otimes \mathbb{C}$, which then maps to an element $\Psi_{f}$ of $M_{!, B} \otimes \mathbb{C}$. In fact $\psi_{f}$ and $\Psi_{f}$ are killed by $\Phi_{f}$. The next lemma was used implicitly in [Dummigan et al. 2003, Section 5].

Lemma 7.2. Under the comparison (de Rham) isomorphism $M_{!, \mathrm{dR}} \otimes \mathbb{C} \rightarrow M_{!, B} \otimes \mathbb{C}$, the image of $f \in \mathrm{Fil}^{k-1} M_{f, \mathrm{dR}}$ is $\Psi_{f}$.

We sketch the reason. As on the line preceding [Diamond et al. 2004, (4)], $f$ corresponds to a differential $(2 \pi i)^{k-1} f(\tau) d z^{\otimes k-2} d \tau$ on $\Gamma_{1}(N) \backslash \mathfrak{H}$, with coefficients in a certain local system with fibres $\operatorname{Sym}^{k-2} H_{\mathrm{dR}}^{1}\left(E_{\tau}\right)$, where $E_{\tau}=\mathbb{C} /\langle 1, \tau\rangle_{\mathbb{Z}}$. In the cohomology of $E_{\tau}$, the class of $d z$ may be identified with $\tau X+Y$, where $X$ and $Y$ are certain generators for the integral cohomology of $E_{\tau}$. Standard arguments show that the comparison map defined in [Diamond et al. 2004, 1.2.4] (in terms of resolving a locally constant sheaf) is effected via integration along chains in $\Gamma_{1}(N) \backslash \mathfrak{H}$.

Theorem 7.3. For $f$ and $\lambda$ as above, suppose that $\lambda$ is not a congruence prime for $f$ in $S_{k}\left(\Gamma_{1}(N), \chi\right)$. Then

$$
\operatorname{ord}_{\lambda}\left(\frac{L_{f}(k-1)}{(2 \pi i)^{k-1} \Omega^{\kappa(k-1)}}\right)<0 .
$$

Proof. Let $\Phi_{f}$ be a generator of $\operatorname{Symb}_{k}\left(\Gamma_{1}(N), \chi, O_{(\lambda)}\right)^{\kappa(k)}\left[\mathscr{I}_{f}\right]$. (Tensoring with $O_{\lambda}$, this can be viewed as the same $\Phi_{f}$ in Lemma 6.2.) Let $\theta_{f}$ be a generator for $\mathfrak{M}_{f, B}^{k(k)} \otimes O_{(\lambda)}$. Say that $\Phi_{f}$ maps to $b \theta_{f}$ under the natural map. Say also that $\Psi_{f}^{\kappa(k)}=c \Phi_{f}$. Then, using Lemma 7.2, $f \in \mathrm{Fil}^{k-1} \mathfrak{M}_{f, \mathrm{dR}}$ maps to $b c \theta_{f}$, and so by definition, $\omega^{\kappa(k)}=b c$. By Lemma 7.1, $(2 \pi i)^{k-1} \Omega^{\kappa(k-1)}=b c$ up to a unit in $O_{(\lambda)}$.

The coefficient of $X^{k-2}$ in $\Psi_{f}^{k(k)}(\{\infty\}-\{0\})$ is $(2 \pi i)^{k-1} \int_{0}^{i \infty} f(\tau) \tau^{k-2} d \tau=$ $\Gamma(k-1) L_{f}(k-1)$. Since $\ell>k-2$, the factor of $\Gamma(k-1)$ does not matter. By Lemma 6.2, the coefficient of $X^{k-2}$ in $\Phi_{f}(\{\infty\}-\{0\})$ is congruent to $1(\bmod \lambda)$, 
since $\Phi_{\chi}(\{\infty\})=X^{k-2}$ and $\Phi_{\chi}(\{0\})=0$. Hence $L_{f}(k-1)=c u$ for some unit $u \in O_{(\lambda)}$. We find now that

$$
\frac{L_{f}(k-1)}{(2 \pi i)^{k-1} \Omega^{\kappa(k-1)}}=\frac{c u}{b c}=\frac{u}{b},
$$

so it suffices to prove that $\operatorname{ord}_{\lambda}(b)>0$. But this is a direct consequence of Lemma 6.2, given that $\Phi_{\chi} \in \operatorname{Bound}_{k}\left(\Gamma_{1}(N), \chi, O_{(\lambda)}\right)$, which is the kernel of the map from $\operatorname{Symb}_{k}\left(\Gamma_{1}(N), \chi, O_{(\lambda)}\right)$ to $\mathfrak{M}_{!, B} \otimes O_{(\lambda)}$.

\section{Failure of the congruence prime condition}

Suppose that $\lambda$ is a congruence prime for $f$ in $S_{k}\left(\Gamma_{1}(N), \chi\right)$. Then (if we make $K$ big enough) there is another newform $g$ such that $f \equiv g(\bmod \lambda)$. Let $\rho_{f}$ and $\rho_{g}$ be the $\lambda$-adic realisations of $\mathfrak{M}_{f}$ and $\mathfrak{M}_{g}$, considered as representations of $\mathrm{Gal}(\overline{\mathbb{Q}} / \mathbb{Q})$. The reductions $\bar{\rho}_{f}$ and $\bar{\rho}_{g}$ are both extensions of $\mathbb{F}_{\lambda}\left(\chi^{-1}\right)$ by $\mathbb{F}_{\lambda}(1-k)$. Unlike the irreducible case, we cannot be sure that they are isomorphic, but it is conceivable that it could sometimes happen, for example, if $\operatorname{dim}\left(\mathfrak{M}_{!, \lambda} / \lambda \mathfrak{M}_{!, \lambda}\right)[\mathfrak{m}]=2$. Let us consider the case $\bar{\rho}_{f} \simeq \bar{\rho}_{g}$. Then $\rho_{f}$ and $\rho_{g}$ are both deformations of $\bar{\rho}_{f}$. Note that the space of $\bar{\rho}_{f}$ is $A[\lambda]$. Let $r$ be minimal such that $\rho_{f}$ and $\rho_{g}$ are different $\left(\bmod \lambda^{r+1}\right)$. Then

$$
\rho_{g}(\sigma) \equiv \rho_{f}(\sigma)\left(I+\lambda^{r}(\theta(\sigma))\right) \quad\left(\bmod \lambda^{r+1}\right)
$$

defines a cocycle $\theta$ of $\mathrm{Gal}(\overline{\mathbb{Q}} / \mathbb{Q})$, representing a nonzero cohomology class $[\theta] \in$ $H^{1}\left(\mathbb{Q}, \operatorname{ad}^{0}\left(\bar{\rho}_{f}\right)\right)$.

Bearing in mind the composition series for $A[\lambda]$, we have an exact sequence of $\mathbb{F}_{\lambda}[\mathrm{Gal}(\overline{\mathbb{Q}} / \mathbb{Q})]$-modules:

$$
0 \longrightarrow \mathbb{F}_{\lambda}(\chi, 1-k) \longrightarrow \operatorname{ad}^{0}\left(\bar{\rho}_{f}\right) \stackrel{\pi}{\longrightarrow} A[\lambda](k-1) \longrightarrow 0 .
$$

The projection $\pi$ is evaluation on a generator of the submodule $\mathbb{F}_{\lambda}(1-k)$. This gives us $\pi_{*}[\theta] \in H^{1}(\mathbb{Q}, A[\lambda](k-1))$. Without going into laborious detail, it is plausible that sometimes this might give us a nonzero element of the Selmer group $H_{f}^{1}\left(\mathbb{Q}, A_{\lambda}(k-1)\right)$. By finiteness of this Selmer group [Kato 2004], this would give a nonzero element of $\lambda$-torsion in $\amalg(k-1)$. In (1) (for $j=k-1$ ), this could cancel the contribution from $\# H^{0}(\mathbb{Q}, A(k-1))$, making it unnecessary for $\lambda$ to occur in the denominator of $L_{f}(k-1) /\left((2 \pi i)^{k-1} \Omega^{\kappa(k-1)}\right)$.

\section{References}

[Ash and Stevens 1986] A. Ash and G. Stevens, "Modular forms in characteristic $l$ and special values of their L-functions", Duke Math. J. 53:3 (1986), 849-868. MR 88h:11036 Zbl 0618.10026 
[Bloch and Kato 1990] S. Bloch and K. Kato, "L-functions and Tamagawa numbers of motives", pp. 333-400 in The Grothendieck Festschrift, Vol. I, edited by P. Cartier et al., Progr. Math. 86, Birkhäuser, Boston, 1990. MR 92g:11063 Zbl 0768.14001

[Carayol 1986] H. Carayol, "Sur les représentations $l$-adiques associées aux formes modulaires de Hilbert”, Ann. Sci. École Norm. Sup. (4) 19:3 (1986), 409-468. MR 89c:11083 Zbl 0616.10025

[Deligne 1979] P. Deligne, "Valeurs de fonctions $L$ et périodes d'intégrales", pp. 313-346 in Automorphic forms, representations and L-functions (Corvallis, OR, 1977), edited by A. Borel and W. Casselman, Proc. Sympos. Pure Math., XXXIII, Amer. Math. Soc., Providence, R.I., 1979. With an appendix by N. Koblitz and A. Ogus. MR 81d:12009 Zbl 0449.10022

[Deligne and Serre 1974] P. Deligne and J.-P. Serre, "Formes modulaires de poids 1", Ann. Sci. École Norm. Sup. (4) 7 (1974), 507-530 (1975). MR 52 \#284 Zbl 0321.10026

[Diamond and Shurman 2005] F. Diamond and J. Shurman, A first course in modular forms, Graduate Texts in Mathematics 228, Springer, New York, 2005. MR 2006f:11045 Zbl 1062.11022

[Diamond et al. 2004] F. Diamond, M. Flach, and L. Guo, "The Tamagawa number conjecture of adjoint motives of modular forms”, Ann. Sci. École Norm. Sup. (4) 37:5 (2004), 663-727. MR 2006e:11089 Zbl 1121.11045

[Dummigan 2000] N. Dummigan, "Period ratios of modular forms", Math. Ann. 318:3 (2000), 621636. MR 2002a:11049 Zbl 1041.11037

[Dummigan 2005] N. Dummigan, "Rational torsion on optimal curves", Int. J. Number Theory 1:4 (2005), 513-531. MR 2007f:11064 Zbl 02244187

[Dummigan et al. 2003] N. Dummigan, W. Stein, and M. Watkins, "Constructing elements in Shafarevich-Tate groups of modular motives", pp. 91-118 in Number theory and algebraic geometry, edited by M. Reid and A. Skorobogatov, London Math. Soc. Lecture Note Ser. 303, Cambridge Univ. Press, Cambridge, 2003. MR 2005g:11071 Zbl 02150712

[Edixhoven 1997] B. Edixhoven, "Serre's conjecture", pp. 209-242 in Modular forms and Fermat's last theorem (Boston, 1995), edited by G. Cornell et al., Springer, New York, 1997. MR 1638480 Zbl 0918.11023

[Faltings and Jordan 1995] G. Faltings and B. W. Jordan, "Crystalline cohomology and GL(2, Q)", Israel J. Math. 90:1-3 (1995), 1-66. MR 96g:11055 Zbl 0854.14010

[Greenberg and Stevens 1993] R. Greenberg and G. Stevens, " $p$-adic $L$-functions and $p$-adic periods of modular forms”, Invent. Math. 111:2 (1993), 407-447. MR 93m:11054 Zbl 0778.11034

[Kato 2004] K. Kato, " $p$-adic Hodge theory and values of zeta functions of modular forms", Astérisque 295 (2004), 117-290. Cohomologies $p$-adiques et applications arithmétiques. III. MR 2006b: 11051 Zbl 02123616

[Katz 1973] N. M. Katz, " $p$-adic properties of modular schemes and modular forms”, pp. 69-190 in Modular functions of one variable, III (Antwerp, 1972), edited by W. Kuyk and J.-P. Serre, Lecture Notes in Mathematics 350, Springer, Berlin, 1973. MR 56 \#5434 Zbl 0271.10033

[Kohnen and Zagier 1984] W. Kohnen and D. Zagier, "Modular forms with rational periods", pp. 197-249 in Modular forms (Durham, 1983), edited by R. A. Rankin, Ellis Horwood Ser. Math. Appl.: Statist. Oper. Res., Horwood, Chichester, 1984. MR 87h:11043 Zbl 0618.10019

[Manin 1973] J. I. Manin, "Periods of cusp forms, and p-adic Hecke series", Mat. Sb. (N.S.) 92(134) (1973), 378-401, 503. MR 49 \#10638 Zbl 0293.14007

[Ribet 1976] K. A. Ribet, "A modular construction of unramified $p$-extensions of $\left(\mu_{p}\right)$ ", Invent. Math. 34:3 (1976), 151-162. MR 54 \#7424 Zbl 0338.12003

[Scholl 1990] A. J. Scholl, "Motives for modular forms”, Invent. Math. 100:2 (1990), 419-430. MR 91e:11054 Zbl 0760.14002 
[Stein $\geq 2008]$ W. Stein, "Rationals part of the special values of the $L$-functions of level 1", Database, Available at http://modular.fas.harvard.edu/Tables/lratios_level1.html.

Received January 10, 2007.

NeIL DUMMigan

UNIVERSITY OF SHEFFIELD

Department of PuRe Mathematics

HICKS BUILDING

HOUNSFIELD ROAD

SHEFFIELD, S3 7RH

UNITED KINGDOM

n.p.dummigan@shef.ac.uk

http://www.neil-dummigan.staff.shef.ac.uk/ 